work. In an average of four pages per paper (as here) it is possible to cover only the main points and to give few references, which rarely seem to include the author himself. Less modesty and more pointers to further information would be helpful.

Cavendish Laboratory

V. E. Cosslett

Cambridge, England

\section{Transmission Electron Microscopy of Metals.}

By G. Thomas. Pp. xiv +299 . New York; London: Wiley, 1962. Price 87s.

In the early days of electron microscopy it was possible to examine metals only by taking plastic replicas of their surfaces. In the past five or six years, however, controlled etching techniques have been developed which allow films of almost any metal or alloy to be prepared thin enough for electron microscopy at $100 \mathrm{kV}$., i.e. less than about $0 \cdot 3 \mu$ in thickness. Coming as it did soon after the establishment of the dislocation theory of metals, this sparked off an almost explosive outburst of activity in applying the electron microscope to problems in metal physics and metallurgy. Although there have been one or two reviews of the results so obtained, the present text is the first to describe in detail the subject as a whole. The author is well qualified to do so, having been in the middle of developments first in Cambridge and now in the University of California.

$\mathrm{He}$ sets out to provide a working handbook for those new to the electron microscopy of metals, giving principles as well as recipes. The first chapter is an introduction to the interaction of electrons with matter, with particular attention to the theory of diffraction, and Chapter 2 applies this knowledge in explaining the mechanism of image formation and contrast from crystalline specimens. For those with a knowledge of crystallography and $\mathrm{X}$-ray diffraction, prerequisites for the course at Berkeley on which the book is based, all this will no doubt be plain sailing. But anyone new to the subject will probably need to make full use of the references given to more detailed treatments of these topics. Similarly, a treatment of the principles, construction and operation of the electron microscope in 50 pages can be no more than an outline introduction, to be supplemented by further reading. The remaining half of the book, Chapters 4 and 5, describes methods of specimen preparation and reviews the results obtained with them. This part is very well done, and it will probably be a revelation to many metallographers. The detailed evidence of dislocations and other defects in metals is fully set out, with many micrographs. The recent work on dynamic phenomena, such as phase transformations in alloys, is probably even less known and, being the author's special area of interest, is clearly and concisely treated.

For the newcomer to the electron microscopy of metals, the Appendices may well prove as valuable as the body of the text. Appendix A describes in detail the steps in analysing an electron micrograph of a thin aluminium foil and the selected-area diffraction pattern obtained from it: determination of foil orientation, slip planes and foil thickness. Appendix $\mathrm{C}$ is a short review of the main bibliographical sources for electron microscopy in general, and Appendix $D$ outlines the requirements of an electron-microscopical laboratory. Appendix $\mathbf{E}$ lists the latest values of atomic scattering factors for the elements.

The text is clearly written and illustrated with a wealth of line diagrams and micrographs, 159 in all. The details in most of the latter would have shown up better if reproduced on art paper instead of in the text, but doubtless to do so would have added appreciably to the cost. This is a minor criticism, and the book can be recommended to all who wish to learn of the great advances which the electron microscope has brought in our knowledge of the interior structure of metals. To those who are setting out to use the new methods, it must be an indispensable guide and mentor. The chief omission, to one of a cautious turn of mind, is of any critical discussion (apart from a few lines on p. 188) of the relevance of the observations made on thin films to our understanding of the properties of metals in bulk. Now that the electron microscope is being increasingly used as a metallurgical laboratory in miniature, with heating, cooling, straining and even reaction of the specimen during observation, the limitations as well as advantages of the method need to be kept fully in mind. However, the author may feel that the occupational scepticism of the trained metallographer will provide a natural counterbalance to his own evident enthusiasm for his subject.

\section{Cavendish Laboratory}

V. E. Cosslett

England 